\title{
The integration of bevacizumab improves tumor response and survival in patients with refractory cervical cancer treated with radical chemoradiotherapy
}

\author{
Hua Yang ${ }^{1 \#}$, Ying Zhang ${ }^{1 "}$, Changhao Liu ${ }^{1}$, Bin Feng ${ }^{1}$, Jianjun Zhang ${ }^{2}$, Yan Zhou ${ }^{1}$, Yutian Yin ${ }^{1}$, \\ Jianping $\mathrm{Li}^{1}$, Weiwei $\mathrm{Li}^{1}$, Vincent Balaya ${ }^{3}$, Mei Shi ${ }^{1}$, Lina Zhao ${ }^{1}$, Lichun Wei ${ }^{1}$ \\ ${ }^{1}$ Department of Radiation Oncology, First Affiliated Hospital of Air Force Medical University, Xi'an, China; ${ }^{2}$ Department of Obstetrics and \\ Gynecology, West China Second University Hospital, Sichuan University, Chengdu, China; ${ }^{3}$ Département Femme-Mère-Enfant, Service de \\ Gynécologie, Centre Hospitalier Universitaire Vaudois, Lausanne, Switzerland \\ Contributions: (I) Conception and design: H Yang, Y Zhang, L Wei; (II) Administrative support: C Liu, B Feng, M Shi, L Zhao, V Balaya; (III) \\ Provision of study materials or patients: J Li, L Wei, J Zhao, H Yang; (IV) Collection and assembly of data: H Yang, Y Zhang, C Liu, W Li; (V) Data \\ analysis and interpretation: Y Yin, Y Zhou; (VI) Manuscript writing: All authors; (VII) Final approval of manuscript: All authors. \\ "These authors contributed equally to this work. \\ Correspondence to: Mei Shi. Department of Radiation Oncology, First Affiliated Hospital of Air Force Medical University, Xi'an 710032, Shaanxi, \\ China. Email: mshi82@fmmu.edu.cn; Lina Zhao. Department of Radiation Oncology, First Affiliated Hospital of Air Force Medical University, Xi'an \\ 710032, Shaanxi, China. zhaolina@fmmu.edu.cn; Lichun Wei. Department of Radiation Oncology, First Affiliated Hospital of Air Force Medical \\ University, Xi'an 710032, Shaanxi, China. weilichun2020603@163.com.
}

Background: Management of refractory cervical cancers (CC) is a debated question and rises dilemma in clinical practice. This study aimed to assess the safety and effectiveness of bevacizumab combined with concurrent chemoradiotherapy in the treatment of refractory CC.

Methods: A total of 129 patients with refractory CC who received radical concurrent chemoradiotherapy (CCRT) were included in this study. Among the patients, 64 received combination treatment with bevacizumab, while the 65 remaining patients did not receive bevacizumab. Treatment response was evaluated according to the Response Evaluation Criteria in Solid Tumorsversion1.1. The Cox proportionalhazards model was applied to determine prognostic factors associated with overall survival and the tumor response during treatment was analyzed for patients treated with bevacizumab.

Results: Bevacizumab was an independent prognostic factor $(\mathrm{P}=0.017)$. Therefore, we only analyzed 64 patients who received combination treatment with bevacizumab. In the 64 patients treated with bevacizumab, the 3-year OS, locoregional relapse-free survival, and distant metastasis-free survival rates were $87.2 \%, 98.1 \%$, and $81 \%$, respectively. Complete clinical response rates were $37.8 \%(17 / 45)$ for patients with neoadjuvant chemotherapy and chemoradiotherapy after neoadjuvant chemotherapy (NACT), complete clinical response rates were 62.5\% (40/64), 73.3\% (33/45) and 52.6\% (10/19) before brachytherapy (BT), respectively for the entire cohort, patients with NACT and chemoradiotherapy and patients with chemoradiotherapy only. The 2-year OS rate was higher for patients who achieved a complete clinical response $\mathrm{BT}$ than for patients who did not, $94.6 \%$ vs. $73.2 \%, \mathrm{P}=0.03$. Among the 64 patients who received it, 28 (43.8\%) experienced hematological toxicities of grade 3 or 4 , and $3(4.7 \%)$ experienced grade 3 gastrointestinal toxicities.

Conclusions: Bevacizumab combined with radical chemoradiotherapy is a safe and tolerable treatment option for refractory CC, with quicker tumor regression and high OS, locoregional relapse-free survival, and distant metastasis-free survival rates.

Keywords: bevacizumab; chemoradiotherapy; refractory cervical cancer. 
Submitted May 18, 2021. Accepted for publication Jul 22, 2021.

doi: 10.21037/atm-21-3521

View this article at: https://dx.doi.org/10.21037/atm-21-3521

\section{Introduction}

Cervical cancers (CC) with bulky primary tumor, regional and distant lymph node metastasis, and huge pelvic relapse after radical surgery have a poor prognosis and are widely considered as refractory. Patients with refractory CC are usually eligible for concurrent chemoradiotherapy (CCRT) even those with multiple risk factors indicating poor survival. According to international guidelines, Bevacizumab is a targeted therapy that was recommended as a first-line treatment for incurable CC in $2015(1,2)$; however, targeted therapies have not been adopted with curative intent for patients with refractory CC.

Several studies have shown that patients with a primary tumor larger than $5 \mathrm{~cm}$ and/or non-squamous pathology, have lower local control and survival rates due to low response to CCRT, and large high-risk clinical target volume for brachytherapy (BT) (3-9). The 5-year survival rates for patients with IIIC1 and IIIC2 of the 2018 FIGO classification (International Federation of Gynecology and Obstetrics) were reported as $60.8 \%$ and $37.5 \%$, respectively (10). Recently, Ferenschild et al. reported a 5 -year overall survival (OS) rate of $45 \%$ for CC patients with pelvic failure after salvage pelvic exenteration surgery (11).

Our previous research highlighted that high vascular endothelial growth factor (VEGF)/VEGF receptor expression was associated with a poor outcome in CC (12). Bevacizumab, an angiogenesis inhibitor, inhibits tumor angiogenesis by blocking the function of VEGF. The GOG240 phase III trial concluded that chemotherapy combined with bevacizumab extended the survival of patients with recurrent and metastatic CC (2). RTOG 0417 trial is the first and the only trial used bevacizumab in LACC patients' initial treatment, but with many limitations (13). Most patients included in 0417 trial did not meet the "refractory" criteria (e.g., tumor size, lymphatic metastasis); Bevacizumab was only used in concurrent chemoradiation instead of in NACT, which had already been proven to be effective in reducing tumor volume. However, literature remains poor about the impact of immunotherapy such as bevacizumab in case of refractory CC. The adjunction of bevacizumab to CCRT in this setting has to be assessed to determine whether local tumor response is improved so as patients' oncologic outcomes.
This study aimed to assess the impact of bevacizumab combined with CCRT in the treatment of refractory CC. We present the following article in accordance with the STROBE reporting checklist (available at https://dx.doi. org/10.21037/atm-21-3521).

\section{Methods}

\section{Population study}

Patients with CC managed in two Chinese tertiary hospitals between January 2016 and December 2019 were screened. For this study, inclusion criteria were as following: (I) a diagnosis of squamous carcinoma, adenocarcinoma, or adenosquamous carcinoma of the uterine cervix; (II) FIGO stage (2018) IIB to IIIC2, or IVB with curable distant lymph node metastasis other than distant organ involvement, or huge pelvic relapse (tumor diameter $>5 \mathrm{~cm}$ ) after radical surgical treatment; (III) Zubrod performance status score of 0 to 2 ; and (IV) hemoglobin $\geq 10 \mathrm{~g} / \mathrm{L}$ (transfusion allowed to meet this criterion). Patients were required to complete pelvic magnetic resonance imaging (MRI) and computed tomography (CT) scans of the chest, abdomen, and pelvis to assess the tumor response to treatment. Patients with contraindications to bevacizumab such as significant bleeding or thromboembolic events within the previous 6 months were excluded.

The study was conducted in adherence with the guidelines of the Declaration of Helsinki (as revised in 2013). This study was approved by the Institutional Review Board of the First Affiliated Hospital of Fourth Military Medical University (protocol code: KY20162017-2). Written informed consent to publish this paper was obtained from the study participants.

\section{Radiotherapy and concurrent chemotherapy}

A pelvic and abdominal cavity enhanced scan was performed using a CT simulator (Brilliance CT, Big Bore), with a $5 \mathrm{~mm}$-thick slices and scanning range from the superior end of the kidneys to $5 \mathrm{~cm}$ inferior to the ischial tuberosity. The external beam radiotherapy (EBRT) dose was 45-50 Gy/25 fractions for the pelvis and 62.5 Gy/25 fractions for metastatic lymph nodes, and was delivered using 
the volumetric modulated arc therapy or three-dimensional conformal radiotherapy technique. The dose limits for organs at risk were based on the report by Mell et al. (14). Irradiation was performed using a $6 \mathrm{MV} \mathrm{X}$-ray beam from the Varian Clinac IX linear accelerator. CT image-guided BT was conducted using a NucletronMicro Selectron-HDR Ir-192 remote BT instrument, with 1-2 fractions/week up to a total of 4-5 fractions. All patients received concurrent chemotherapy with cisplatin (DDP) $40 \mathrm{mg} / \mathrm{m}^{2}$ weekly or docetaxel $75 \mathrm{mg} / \mathrm{m}^{2}$ and cisplatin $75 \mathrm{mg} / \mathrm{m}^{2}$ tri-weekly.

\section{Bevacizumab treatment}

Bevacizumab was administered at $7.5 \mathrm{mg} / \mathrm{kg}$ tri-weekly for both the neoadjuvant and concurrent regimens. The NACT regimen consisted of docetaxel $75 \mathrm{mg} / \mathrm{m}^{2}$ and cisplatin $75 \mathrm{mg} / \mathrm{m}^{2}$ tri-weekly. The CCRT regimen consisted of DDP delivered at $75 \mathrm{mg} / \mathrm{m}^{2}$ tri-weekly.

\section{Adverse events and follow-up}

Severity of hematologic and digestive toxicities were assessed according to the Radiation Therapy Oncology Group/ European Organization for Research and Treatment of Cancer classification criteria for radiation damage. Other acute and late side effects were graded using the Common Terminology Criteria for Adverse Eventsversion3.0. All patients were followed up regularly from the end of radiotherapy. The patients were initially followed up every 3 months in the first year after treatment, at 6-month intervals in the second and the third years, and once per year thereafter.

\section{Data management and statistical analysis}

Tumor size was measured on T2MRI images. Tumor volume was calculated as length $\times$ width $\times$ height $\times \pi / 6(15)$. The residual tumor volume percentage was calculated as the residual tumor volume before EBRT or BT divided by the tumor volume before treatment. The tumor regression rate was 1-residual tumor volume percentage. Complete response (CR) was defined as the complete disappearance of the tumor on T2 MRI scans according to Response Evaluation Criteria in Solid Tumorsversion1.1. The OS, local relapse-free survival (LRFS), and distant metastasisfree survival (DMFS) rates were calculated.

The Cox proportional-hazards model was applied to determine prognostic factors associated with overall survival. Variables yielding $\mathrm{P}$ values lower than 0.05 by univariate analysis were entered into a multivariate Cox proportional hazards regression model to determine variables independently associated with OS.

Overall survival and free-recurrence survival were analysed by using Kaplan-Meier analysis and compared by applying log-rank test.

Statistical analyses were performed using SPSS 22.0 (SPSS Inc., Chicago, IL, USA).

\section{Results}

\section{Patient characteristics}

Between January 2016 and December 2019, 129 patients with refractory $\mathrm{CC}$ from our institution met the eligibility criteria. Among these 129 patients, 64 received combination treatment with bevacizumab, while the 65 remaining patients did not receive bevacizumab.

The characteristics of these patients and a Cox proportional-hazards analysis of their OS are summarized in Table S1. Univariate and multivariate analyses revealed bevacizumab to be an independent prognostic factor for patients with refractory CC treated with radical chemoradiotherapy $(\mathrm{P}=0.017)$. Therefore, of the 129 patients treated with radical chemoradiotherapy, the 64 patients who received combination treatment with bevacizumab were included in further analyses.

The median age of patients at diagnosis was 50 years (range, 20-67 years). The median diameter of the primary tumor was $6.8 \mathrm{~cm}$ (range, $5-11.5 \mathrm{~cm}$ ). Among the patients, 48 (75\%) were at FIGO stage IIB-IIIC2 (2018), 10 (15.6\%) had stage IVB disease with distant lymphatic metastasis, and $6(9.4 \%)$ had recurrent disease with bulky lesions. Positive lymph nodes were present in 43 cases (67.2\%), 20 of which were para-aortic lymph node metastasis. Squamous cell carcinoma was the predominant pathological subtype (58/64, 90.6\%). Of the study participants, 19 received bevacizumab and CCRT, while the other 45 patients received 2 cycles of bevacizumab with NACT before bevacizumab and CCRT due to having a tumor diameter $>6 \mathrm{~cm}, \geq 3$ metastatic pelvic lymph nodes, or distant metastatic lymph nodes. Patient characteristics and treatment details are presented in Table 1 .

\section{Treatment compliance}

All patients completed the planned course of chemoradiation treatment. The median treatment duration was 59.2 (range, 
Table 1 Baseline characteristics of 64 patients treated with a combination of bevacizumabandradical chemoradiotherapy

\begin{tabular}{lc}
\hline Characteristic & $\mathrm{N}(\%)$ \\
\hline Age (range), years & $50[20-67]$ \\
Tumor diameter $(\mathrm{cm})$ & \\
$5-6$ & $28(43.8)$ \\
$\geq 6$ & $36(56.2)$ \\
Tumor volume $\mathrm{M}$ (range) $\mathrm{cm}^{3}$ & $61.3(25.19-264.8)$
\end{tabular}

Tumor volume M (range), $\mathrm{cm}^{3}$

$61.3(25.19-264.8)$

No. of lymph nodes

$$
\begin{aligned}
& \geq 3 \\
& 1-2 \\
& 0 \\
& \text { Ln volume M (range), } \mathrm{cm}^{3} \\
& 2018 \text { FIGO stage } \\
& \text { IIB-IIIC } \\
& \text { IVB-Ln only } \\
& \text { Pelvic relapse after surgery }(\mathrm{d}>5 \mathrm{~cm}) \\
& \text { Radiotherapy technology } \\
& \text { 3DRT } \\
& \text { IMRT }
\end{aligned}
$$

48 (75.0)$$
6(9.4)
$$

Treatment modality

$$
\begin{array}{lc}
\text { NACT+CCRT } & 45(70.3) \\
\text { CCRT only } & 19(29.7) \\
\text { Pathological type } & \\
\text { Squamous carcinoma } & 58(90.6) \\
\text { Non-squamous carcinoma } & 6(9.4) \\
\text { Brachytherapy } & \\
\text { D90 for HR-CTV M (range) (Gy) } & 82.9[78-90] \\
\text { D2cc-rectum M (range) (Gy) } & 71.8(66.9-74.8) \\
\text { D2cc-bladder M (range) (Gy) } & 79.1(76-84.5) \\
\text { D2cc-sigmid colon M (range) (Gy) } & 66.6(60.1-73.2) \\
\text { OTT } & 59.2(55.1-65.8)
\end{array}
$$

3DRT, three dimensional conformal radiotherapy; IMRT, intensity-modulated radiation therapy; NACT, neoadjuvant chemotherapy; CCRT, concurrent chemoradiotherapy; HR-CTV, high risk-clinical target volume; OTT, over treatment time.

Table 2 The tumor response and cCR rate after NACT

\begin{tabular}{lc}
\hline Tumor response after NACT & NACT + CCRT (N=45) \\
\hline $\mathrm{cCR}$ & $17(37.8 \%)$ \\
$\geq 75 \%$ & $35(77.8 \%)$ \\
$\geq 50 \%$ & $39(86.7 \%)$ \\
$\geq 30 \%$ & $45(100 \%)$ \\
$<30 \%$ & 0 \\
\hline
\end{tabular}

Table 3 The tumor response and cCR rate before BT

\begin{tabular}{lccc}
\hline $\begin{array}{l}\text { Tumor response } \\
\text { before BT }\end{array}$ & $\begin{array}{c}\text { Total } \\
(\mathrm{N}=64)\end{array}$ & $\begin{array}{c}\text { NACT + CCRT } \\
(\mathrm{N}=45)\end{array}$ & $\begin{array}{c}\text { CCRT only } \\
(\mathrm{N}=19)\end{array}$ \\
\hline cCR & $40(62.5 \%)$ & $33(73.3 \%)$ & $10(52.6 \%)$ \\
$\geq 75 \%$ & $63(98.4 \%)$ & $45(100 \%)$ & $18(94.7 \%)$ \\
$\geq 50 \%$ & $64(100 \%)$ & $45(100 \%)$ & $19(100 \%)$ \\
$\geq 30 \%$ & $64(100 \%)$ & $45(100 \%)$ & $19(100 \%)$ \\
$<30 \%$ & 0 & 0 & 0 \\
\hline $\begin{array}{l}\text { cCR, clinical complete response; NAT, neoadjuvant chemotherapy; } \\
\text { BT, brachytherapy; CCRT, concurrent chemoradiotherapy. }\end{array}$
\end{tabular}

55.1-65.8) days. Treatment was delayed for 2 weeks in 4 patients due to the occurrence of grade 3 or 4 hematological toxicities. For patients treated with NAT, the median interval from the end of NAT to the start of RT was 3 weeks (range, 2-4).

\section{Clinical response}

A complete clinical response (cCR) was observed in 37.8\% $(17 / 45)$ of patients at the end of NACT and in $62.5 \%(40 / 64)$ of patients BT. The median tumor volume reduction rates were $90.2 \%$ (range, $35.2-100 \%$ ) after NACT and $100 \%$ (range, 74.1-100\%) before BT. Of the 64 patients, 45 $(70.3 \%)$ had tumor shrinkage $>75 \%$. Detailed data of the tumor response and cCR rates after NAT and before BT are shown in Tables 2,3.

\section{Survival}

The median follow-up was 24.1 months (5.1-55.6 months). 
A
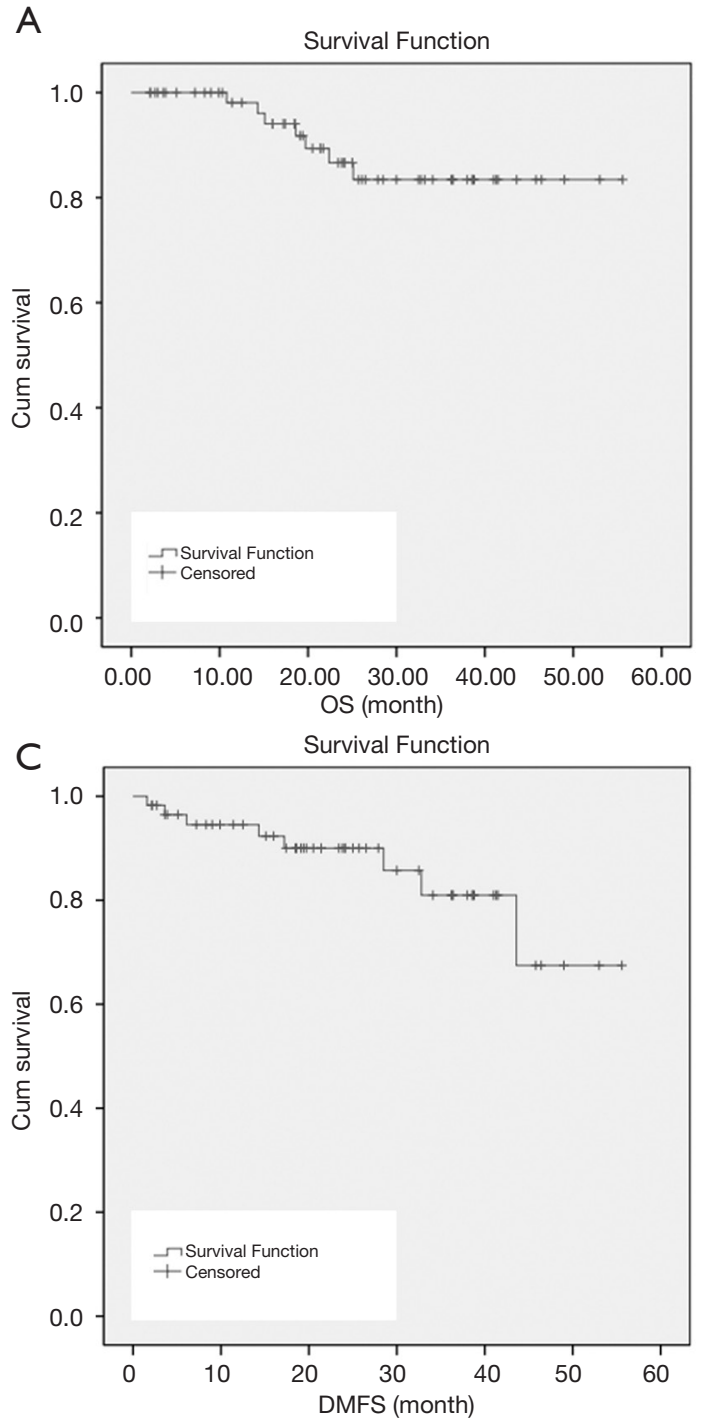

B
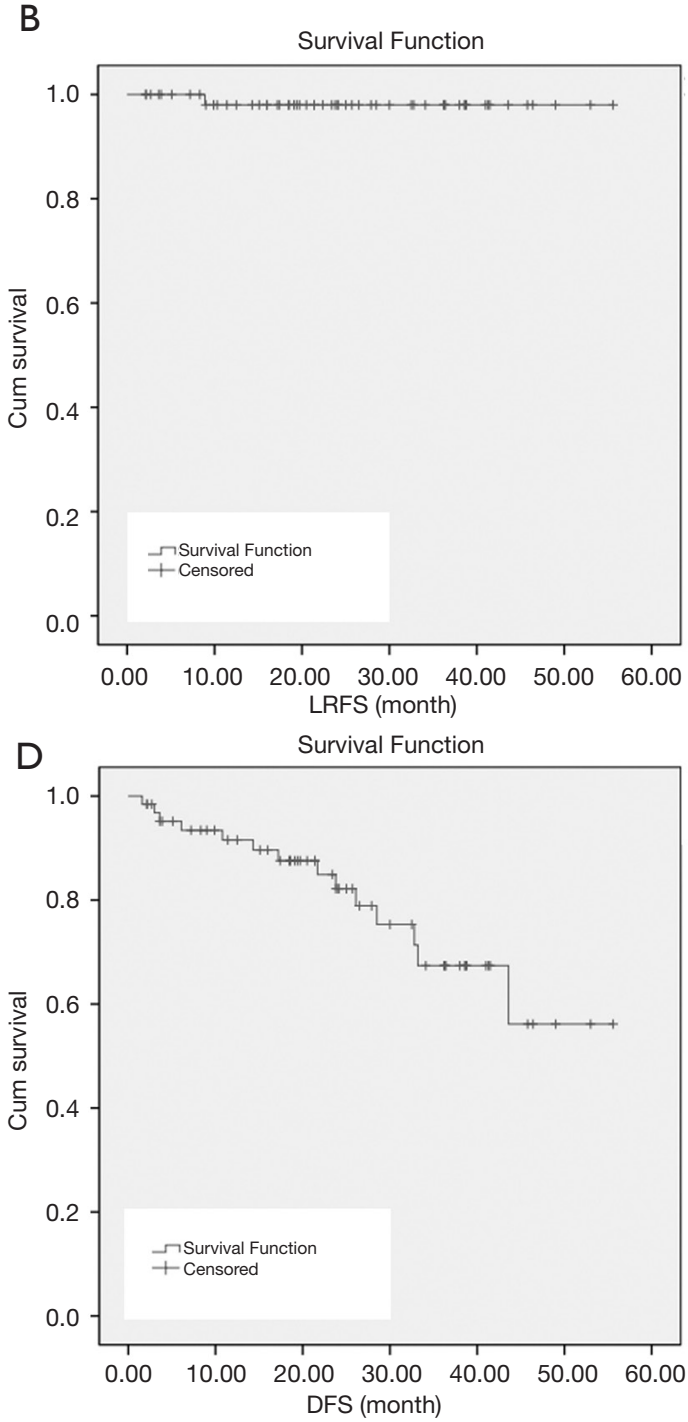

Figure 1 The 3-year clinical survival of 64 patients treated with bevacizumab. (A) OS. (B) LRFS. (C) DMFS. OS, overall survival; LRFS, local relapse-free survival; DMFS, distant metastasis-free survival.

Three patients experienced local regional recurrence, and 6 patients had distant metastasis including 1 with lung metastasis, 2 with bone metastasis, and 3 with supraclavicular lymph node metastasis. Overall, 5 patients died including 1 with local recurrence, 3 with distant metastasis, and 1 with both local recurrence and distant metastasis. The 3-year OS, LRFS, and DMFS rates for all 64 patients were $87.2 \%, 98.1 \%$, and $81 \%$, respectively (Figure 1). Patients with rapid tumor shrinkage showed a better survival outcome than those without. The $1-$ and 2 -year OS rates in patients with cCR were $100 \%$ and
94.6\%, respectively, compared with $100 \%$ and $73.2 \%$ in patients without cCR (Figure 2).

\section{Side effects}

In general, both acute and late toxicities related to bevacizumab were acceptable. Nausea, diarrhea, and neutropenia were the most common acute side effects. The rate of grade 3-4 hematological toxicities was $43.8 \%$ (28/64), and 3 patients (4.7\%) developed acute gastrointestinal toxicities. Grade 2 chronic radiation 


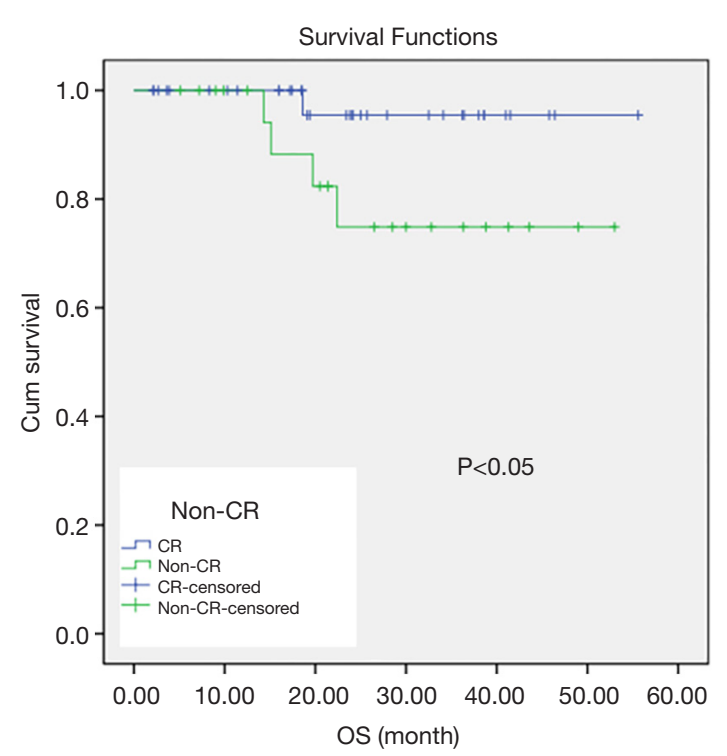

Figure 2 OS was compared between the cCR and non-cCR groups before BT $(\mathrm{P}=0.034)$. OS, overall survival; cCR, clinical complete response; $\mathrm{BT}$, brachytherapy. proctitis occurred in 4 patients $(6.3 \%)$, but no cases of grade 3 or 4 were recorded (Table 4). Additionally, 2 patients (3.1\%) had nosebleed, and 5 (9.4\%) developed grade 1-2 hypertension during bevacizumab treatment; in all of these cases, the patient was cured through symptomatic treatment. No cases of fistula, thrombus, or other bevacizumab-related severe adverse events occurred.

\section{Discussion}

Our research is the first to illustrate that bevacizumab is an independent prognostic factor for patients with refractory CC receiving radical treatment. The 3 -year OS and LRFS of patients with refractory CC who were treated with a combination of bevacizumab and chemoradiotherapy were $87.2 \%$ and $98.1 \%$, respectively. This survival benefit was largely derived from accelerated tumor regression and an increased cCR rate during Pelvic EBRT.

Several adverse prognostic factors for locally advanced CC (LACC) have been reported previously, such as large

Table 4 Acute and chronic side effects in 64 patients treated with a combination of bevacizumab andradical chemoradiotherapy [n (\%)]

\begin{tabular}{|c|c|c|c|c|c|c|}
\hline \multirow{2}{*}{ Events } & \multicolumn{2}{|c|}{ Total $(n=64)$} & \multicolumn{2}{|c|}{$\mathrm{NACT}+\mathrm{CCRT}(\mathrm{n}=45)$} & \multicolumn{2}{|c|}{ CCRT (n=19) } \\
\hline & Grade 1-2 & Grade 3-4 & Grade 1-2 & Grade 3-4 & Grade 1-2 & Grade 3-4 \\
\hline \multicolumn{7}{|l|}{ Acute AEs } \\
\hline Nausea & $32(50)$ & $1(1.6)$ & $20(44.4)$ & $1(2.2)$ & $12(63.2)$ & 0 \\
\hline Diarrhea & $55(85.9)$ & $2(3.1)$ & 40 (88.9) & $1(2.2)$ & $15(78.9)$ & $1(5.3)$ \\
\hline Neutropenia & $36(56.3)$ & $28(43.7)$ & $25(55.6)$ & $20(44.4)$ & $11(57.9)$ & $8(42.1)$ \\
\hline Febrile neutropenia & 0 & $1(1.6)$ & 0 & $1(2.2)$ & 0 & 0 \\
\hline Hypertension & $6(9.4)$ & 0 & $4(8.9)$ & 0 & $2(10.5)$ & 0 \\
\hline Nosebleed & $2(3.1)$ & 0 & $2(4.4)$ & 0 & 0 & 0 \\
\hline Fatigue & $10(15.6)$ & 0 & $7(15.6)$ & 0 & $3(15.8)$ & 0 \\
\hline Urinary & $8(12.5)$ & 0 & $5(11.1)$ & 0 & $3(15.8)$ & 0 \\
\hline \multicolumn{7}{|l|}{ Late Aes } \\
\hline Diarrhea & $3(4.7)$ & 0 & $2(4.4)$ & 0 & $1(5.3)$ & 0 \\
\hline Hematochezia & $4(6.3)$ & 0 & $2(4.4)$ & 0 & $2(10.5)$ & 0 \\
\hline Blood in urine & $3(4.7)$ & 0 & $2(4.4)$ & 0 & $1(5.3)$ & 0 \\
\hline Vaginal vesical fistula & 0 & 0 & 0 & 0 & 0 & 0 \\
\hline Vaginal rectal fistula & 0 & 0 & 0 & 0 & 0 & 0 \\
\hline
\end{tabular}

AE, adverse event; NACT, neoadjuvant chemotherapy; CCRT, concurrent chemoradiotherapy. 
masses, lymph node metastasis, advanced clinical stage, and non-squamous cell carcinomas. Large masses cause hypoxia, decrease tumor sensitivity to local radiotherapy, and increase the risk of lymph node metastasis, resulting in a poor prognosis $(5,6)$. Pötter et al. reported that patients with LACC who had a primary tumor diameter of $>5 \mathrm{~cm}$ had a poor prognosis, with 3-year DFS and OS rates of $70 \%$ and $65 \%$, respectively (16). Accordingly, all participants in our study had a tumor diameter $>5 \mathrm{~cm}$. Among 64 patients, 43 (67.2\%) developed pelvic lymph node metastasis, of which 33 cases $(76.7 \%)$ had $\geq 3$ positive lymph nodes, 20 cases (46.5\%) were accompanied by para-aortic lymph node metastasis (2018 FIGO stage IIIC2), and 13 cases (30.2\%) had lymph node necrosis. Despite the poor prognosis expected for these patients, good survival outcomes were achieved in our study. The results of Cox multivariate analysis suggested that this improvement in survival was associated with the combination of bevacizumab and chemoradiotherapy.

Although the survival benefit and safety of bevacizumab for metastatic and recurrent $\mathrm{CC}$ have been reported in the GOG 240 study and a meta-analysis $(17,18)$, but few reports have focused on the combination of bevacizumab with chemoradiotherapy in LACC, especially in patients with bulky tumor. To date, RTOG 0417 is the only prospective work focused on the efficacy of combining bevacizumab with radiotherapy for LACC (13). In RTOG 0417, bevacizumab was used concomitantly with CCRT to treat 49 cases; the 2-year OS, disease-free survival (DFS), and local-regional control (LRC) were $89.8 \%, 75.1 \%$, and $72.8 \%$, respectively. In the present study, the 3 -year OS, LRFS, and DMFS rates were $87.2 \%, 98.1 \%$, and $81 \%$, despite most patients in our research being at advanced stages (2018 FIGO stage: IIICIVB, $67.1 \%$ ) and having bulky primary tumors (median tumor diameter, $6.8 \mathrm{~cm}$; range, $5-11.5 \mathrm{~cm}$ ).

The VEGF signaling pathway promotes tumor proliferation, enhances tumor-related angiogenesis, and even induces cancer immune tolerance. As a targeted VEGF signaling inhibitor, bevacizumab has been reported to extend patient survival in a variety of solid tumors (19). Previous studies on CC have reported that a huge primary tumor (diameter $>5 \mathrm{~cm}$ ) and a large residual mass after Pelvic EBRT were associated with a higher local failure rate $(20,21)$. Therefore, tumor shrinkage during EBRT closely related to local control and survival.

In our study, the addition of bevacizumab to chemoradiotherapy significantly improved the local mass regression rate, with a median shrinkage rate of $100 \%$ (range, $74.1 \%$ to $100 \%$ ), which was better than the rate of $75.2 \%$ reported for EBRT with concurrent chemotherapy by Carvalho Hde et al. (22). The cCR rates after NACT and before BT were $37.8 \%$ and $62.5 \%$ respectively, which were better than the rates reported in a phase II study by Ferrandina et al. in $2018(11.1 \%$ and $48.8 \%$ after neoadjuvant chemotherapy and CCRT, respectively) (23).

Our study showed an encouraging cCR rate after NACT and EBRT, which facilitated subsequent BT and a more reasonable dose distribution. Our data showed that patients with cCR before BT had a better OS than patients without cCR before BT $(\mathrm{P}<0.05$, Figure 2$)$. Therefore, it is reasonable to infer that due to the addition of bevacizumab, the tumor mass shrank rapidly and cCR was achieved after EBRT, thus improving the local control rate and further translating into a survival benefit for patients with bulky CC. These encouraging results have to be confirmed by further randomized prospective clinical trials.

The main limitation of our study is its retrospective design, which may have resulted in bias. However, due to the paucity of CC refractory cases, a monocentric inclusion might have been difficult. At present, a multicenter, prospective clinical study has been launched by our institution (NCT04138992) to further confirm the results of this study in a larger population of patients. Other limitations include the small sample size and short follow-up time. Nevertheless, in the context of a lack of high-grade clinical evidence, our findings support the use of bevacizumab as a clinical treatment option for refractory CC.

\section{Conclusions}

Bevacizumab combined with CCRT is a safe and tolerable treatment for patients with refractory $\mathrm{CC}$ and can improve their survival. With quick tumor regression, and high OS, LRFS, and DMFS rates, this combined treatment modality has great potential to become a first-line choice in clinical practice.

\section{Acknowledgments}

The authors appreciate the academic support from AME Cervical Cancer Collaborative Group.

Funding: This research received no external funding.

\section{Footnote}

Reporting Checklist: The authors have completed the 
STROBE reporting checklist. Available at https://dx.doi. org/10.21037/atm-21-3521

Data Sharing Statement: Available at https://dx.doi. org/10.21037/atm-21-3521

Conflicts of Interest: All authors have completed the ICMJE uniform disclosure form (available at https://dx.doi. org/10.21037/atm-21-3521). The authors have no conflicts of interest to declare.

Ethical Statement: The authors are accountable for all aspects of the work in ensuring that questions related to the accuracy or integrity of any part of the work are appropriately investigated and resolved. The study was conducted in adherence with the guidelines of the Declaration of Helsinki (as revised in 2013) and was approved by the Institutional Review Board of the First Affiliated Hospital of Fourth Military Medical University (protocol code:KY20162017-2) on 22 November, 2016. Written informed consent to publish this paper was obtained from the study participants.

Open Access Statement: This is an Open Access article distributed in accordance with the Creative Commons Attribution-NonCommercial-NoDerivs 4.0 International License (CC BY-NC-ND 4.0), which permits the noncommercial replication and distribution of the article with the strict proviso that no changes or edits are made and the original work is properly cited (including links to both the formal publication through the relevant DOI and the license). See: https://creativecommons.org/licenses/by-nc-nd/4.0/.

\section{References}

1. Tewari KS, Sill MW, Long HJ 3rd, et al. Improved survival with bevacizumab in advanced cervical cancer. $\mathrm{N}$ Engl J Med 2014;370:734-43.

2. Tewari KS, Sill MW, Penson RT, et al. Bevacizumab for advanced cervical cancer: final overall survival and adverse event analysis of a randomised, controlled, open-label, phase 3 trial (Gynecologic Oncology Group 240). Lancet 2017;390:1654-63.

3. Wang N, Li WW, Li JP, et al. Comparison of concurrent chemoradiotherapy followed by radical surgery and highdose-rate intracavitary brachytherapy: a retrospective study of 240 patients with FIGO stage IIB cervical carcinoma. Onco Targets Ther 2014;7:91-100.
4. Mayr NA, Yuh WT, Zheng J, et al. Tumor size evaluated by pelvic examination compared with 3-D quantitative analysis in the prediction of outcome for cervical cancer. Int J Radiat Oncol Biol Phys 1997;39:395-404.

5. Wu Y, Zhu B, Han J, et al. A comparative dosimetric study of cervical cancer patients with para-aortic lymph node metastasis treated with volumetric modulated arc therapy vs. 9-field intensity-modulated radiation therapy. Ann Transl Med 2019;7:675.

6. Narayan K, Fisher R, Bernshaw D. Significance of tumor volume and corpus uteri invasion in cervical cancer patients treated by radiotherapy. Int J Gynecol Cancer 2006;16:623-30.

7. Pötter R, Tanderup K, Kirisits C, et al. The EMBRACE II study: The outcome and prospect of two decades of evolution within the GEC-ESTRO GYN working group and the EMBRACE studies. Clin Transl Radiat Oncol 2018;9:48-60.

8. Zeng J, Chen J, Zhang D, et al. Assessing cumulative dose distributions in combined external beam radiotherapy and intracavitary brachytherapy for cervical cancer by treatment planning based on deformable image registration. Transl Cancer Res 2020;9:6107-15.

9. Jastaniyah N, Yoshida K, Tanderup K, et al. A volumetric analysis of GTVD and CTVHR as defined by the GEC ESTRO recommendations in FIGO stage IIB and IIIB cervical cancer patients treated with IGABT in a prospective multicentric trial (EMBRACE). Radiother Oncol 2016;120:404-11.

10. Wright JD, Matsuo K, Huang Y, et al. Prognostic Performance of the 2018 International Federation of Gynecology and Obstetrics Cervical Cancer Staging Guidelines. Obstet Gynecol 2019;134:49-57.

11. Mukai Y, Koike I, Matsunaga T, et al. Radiation Therapy for Uterine Cervical Cancer With Lung Metastases Including Oligometastases. In Vivo 2019;33:1677-84.

12. Dang YZ, Zhang Y, Li JP, et al. High VEGFR1/2 expression levels are predictors of poor survival in patients with cervical cancer. Medicine (Baltimore) 2017;96:e5772.

13. Schefter T, Winter K, Kwon JS, et al. RTOG 0417: efficacy of bevacizumab in combination with definitive radiation therapy and cisplatin chemotherapy in untreated patients with locally advanced cervical carcinoma. Int $\mathrm{J}$ Radiat Oncol Biol Phys 2014;88:101-5.

14. Mell LK, Sirák I, Wei L, et al. Bone Marrowsparing Intensity Modulated Radiation Therapy With Concurrent Cisplatin For Stage IB-IVA Cervical Cancer: An International Multicenter Phase II Clinical 
Trial (INTERTECC-2). Int J Radiat Oncol Biol Phys 2017;97:536-45.

15. Tomayko MM, Reynolds CP. Determination of subcutaneous tumor size in athymic (nude) mice. Cancer Chemother Pharmacol 1989;24:148-54.

16. Pötter R, Georg P, Dimopoulos JC, et al. Clinical outcome of protocol based image (MRI) guided adaptive brachytherapy combined with $3 \mathrm{D}$ conformal radiotherapy with or without chemotherapy in patients with locally advanced cervical cancer. Radiother Oncol 2011;100:116-23.

17. Koh WJ, Greer BE, Abu-Rustum NR, et al. Cervical Cancer, Version 2.2015. J Natl Compr Canc Netw 2015;13:395-404; quiz 404.

18. Lim MC, Lee M, Shim SH, et al. Practice guidelines for management of cervical cancer in Korea: a Korean Society of Gynecologic Oncology Consensus Statement. J Gynecol Oncol 2017;28:e22.

19. Garcia J, Hurwitz HI, Sandler AB, et al. Bevacizumab (Avastin ${ }^{\circledR}$ ) in cancer treatment: A review of 15 years of clinical experience and future outlook. Cancer Treat Rev

Cite this article as: Yang H, Zhang Y, Liu C, Feng B, Zhang J, Zhou Y, Yin Y, Li J, Li W, Balaya V, Shi M, Zhao L, Wei L. The integration of bevacizumab improves tumor response and survival in patients with refractory cervical cancer treated with radical chemoradiotherapy. Ann Transl Med 2021;9(14):1184. doi: 10.21037/atm-21-3521
2020;86:102017.

20. Dimopoulos JC, Pötter R, Lang S, et al. Dose-effect relationship for local control of cervical cancer by magnetic resonance image-guided brachytherapy. Radiother Oncol 2009;93:311-5.

21. Tanderup K, Fokdal LU, Sturdza A, et al. Effect of tumor dose, volume and overall treatment time on local control after radiochemotherapy including MRI guided brachytherapy of locally advanced cervical cancer. Radiother Oncol 2016;120:441-6.

22. Carvalho Hde A, Mendez LC, Stuart SR, et al. Implementation of image-guided brachytherapy (IGBT) for patients with uterine cervix cancer: a tumor volume kinetics approach. J Contemp Brachytherapy 2016;8:301-7.

23. Ferrandina G, Palluzzi E, Gallotta V, et al. Neo-adjuvant platinum-based chemotherapy followed by chemoradiation and radical surgery in locally advanced cervical cancer (Lacc) patients: A phase II study. Eur J Surg Oncol 2018;44:1062-8. 


\section{Supplementary}

Table S1 The baseline characteristics of 129 patients with refractory cervical cancer and Cox multivariate analysis of their overall survival

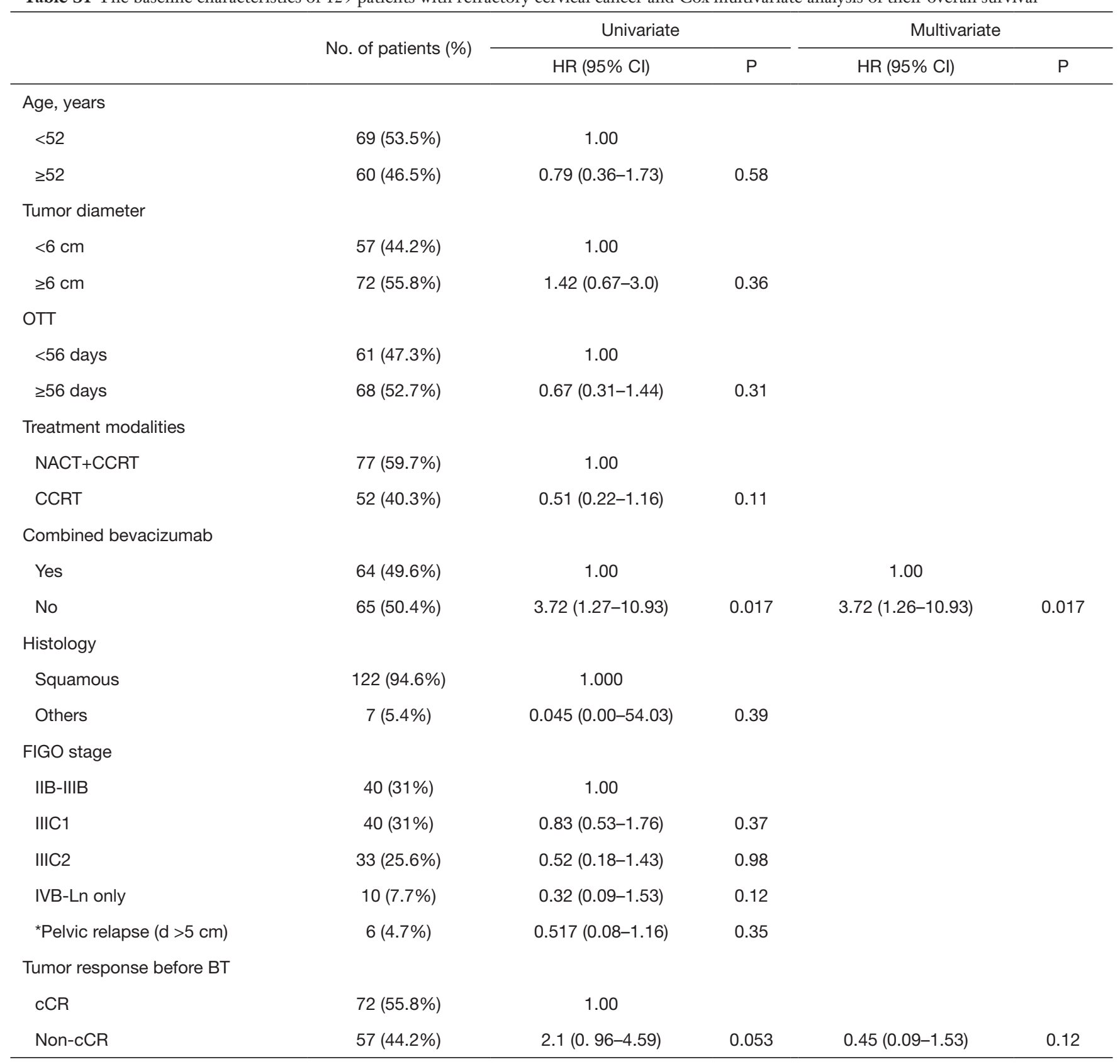

cCR, clinical complete response. 